Supporting Information

\title{
Palladium-Catalyzed Annulation of Phenazastannines with 9- (Dibromomethylene)fluorene and -(thio)xanthenes: Facile Synthesis of Acridine Moiety-Containing Bis(tricyclic) Aromatic Enes
}

\author{
Masaki Shimizu,* Kenta Nishimura, Mizuki Mineyama, and Haruka Fuji
}

Faculty of Molecular Chemistry and Engineering, Kyoto Institute of Technology, 1 Hashikami-cho, Matsugasaki, Sakyo-ku, Kyoto 606-8585, Japan

E-mail:mshimizu@kit.ac.jp

Contents:

Table S1. Screening of Reaction Conditions

NMR Charts of 1a, 2d, 3aa, 3ab, 3ac, 3ad, 3ba, 3bc, and 3bd 
Table S1. Screening of Reaction Conditions ${ }^{a}$

\begin{tabular}{|c|c|c|c|c|c|}
\hline entry & solvent & temp. $\left({ }^{\circ} \mathrm{C}\right)$ & Pd source $(\mathrm{mol} \%)$ & $\mathrm{F}^{-}$source (equiv) & yield $(\%)^{b}$ \\
\hline 1 & 1,4-dioxane & 130 & $\mathrm{Pd}\left(\mathrm{P}^{t} \mathrm{Bu}_{3}\right)_{2}(5.0)$ & $\mathrm{CsF}(5.0)$ & 31 \\
\hline 2 & 1,4-dioxane & 130 & $\mathrm{Pd}\left(\mathrm{PPh}_{3}\right)_{4}(5.0)$ & $\mathrm{CsF}(5.0)$ & 23 \\
\hline 3 & 1,4-dioxane & 130 & Pd-PEPPSI-IPr (5.0) & $\mathrm{CsF}(5.0)$ & 27 \\
\hline 4 & 1,4-dioxane & 130 & SPhos Pd G1 (5.0) & $\mathrm{CsF}(5.0)$ & 24 \\
\hline 5 & 1,4-dioxane & 130 & XPhos Pd G1 (5.0) & $\mathrm{CsF}(5.0)$ & 24 \\
\hline 6 & 1,4-dioxane & 130 & tBuXPhos Pd G1 (5.0) & $\mathrm{CsF}(5.0)$ & 0 \\
\hline 7 & 1,4-dioxane & 130 & BrettPhos Pd G3 (5.0) & $\mathrm{CsF}(5.0)$ & 0 \\
\hline 8 & 1,4-dioxane & 130 & $\mathrm{Pd}_{2} \mathrm{I}_{2}\left(\mathrm{P}^{t} \mathrm{Bu}_{3}\right)_{2}(5.0)$ & $\mathrm{CsF}(5.0)$ & 0 \\
\hline 9 & 1,4-dioxane & 130 & $\mathrm{Pd}(\mathrm{OAc})_{2}(5.0) / \mathrm{P}(o \text {-furyl })_{3}(20)$ & $\mathrm{CsF}(5.0)$ & 31 \\
\hline 10 & 1,4-dioxane & 130 & $\mathrm{Pd}_{2}(\mathrm{dba})_{3}(5.0) / \mathrm{P}(o \text {-furyl })_{3}(20)$ & $\mathrm{CsF}(5.0)$ & 37 \\
\hline 11 & 1,4-dioxane & 130 & $\mathrm{Pd}_{2}(\mathrm{dba})_{3}(5.0) / \mathrm{P}(o \text {-tolyl })_{3}(20)$ & $\mathrm{CsF}(5.0)$ & 35 \\
\hline 12 & 1,4-dioxane & 130 & $\mathrm{Pd}_{2}(\mathrm{dba})_{3}(5.0) / \mathrm{PCy}_{3}(20)$ & $\operatorname{CsF}(5.0)$ & 51 \\
\hline 13 & 1,4-dioxane & 130 & $\mathrm{Pd}_{2}(\mathrm{dba})_{3}(5.0) / \mathrm{PPh}_{3}(20)$ & $\operatorname{CsF}(5.0)$ & 0 \\
\hline 14 & 1,4-dioxane & 130 & $\mathrm{Pd}_{2}(\mathrm{dba})_{3}(5.0) / \mathrm{BINAP}(20)$ & $\mathrm{CsF}(5.0)$ & 15 \\
\hline 15 & 1,4-dioxane & 130 & $\operatorname{Pd}_{2}(\mathrm{dba})_{3}(5.0) /$ xantphos $(10)$ & $\mathrm{CsF}(5.0)$ & 28 \\
\hline 16 & 1,4-dioxane & 130 & $\mathrm{Pd}_{2}(\mathrm{dba})_{3}(5.0) / 1,2-\left(\mathrm{Ph}_{3} \mathrm{P}\right)_{2} \mathrm{C}_{6} \mathrm{H}_{4}(10)$ & $\operatorname{CsF}(5.0)$ & 0 \\
\hline 17 & 1,4-dioxane & 130 & $\mathrm{Pd}_{2}(\mathrm{dba})_{3}(5.0) / \mathrm{dppm}(10)$ & $\mathrm{CsF}(5.0)$ & 0 \\
\hline 18 & 1,4-dioxane & 130 & $\mathrm{Pd}_{2}(\mathrm{dba})_{3}(5.0) /$ dppe $(10)$ & $\mathrm{CsF}(5.0)$ & 19 \\
\hline 19 & 1,4-dioxane & 130 & $\mathrm{Pd}_{2}(\mathrm{dba})_{3}(5.0) / \mathrm{dppp}(10)$ & $\mathrm{CsF}(5.0)$ & 34 \\
\hline 20 & 1,4-dioxane & 130 & $\mathrm{Pd}_{2}(\mathrm{dba})_{3}(5.0) / \mathrm{dppb}(10)$ & $\mathrm{CsF}(5.0)$ & 31 \\
\hline 21 & 1,4-dioxane & 130 & $\mathrm{Pd}_{2}(\mathrm{dba})_{3}(5.0) /$ dpppe $(10)$ & $\mathrm{CsF}(5.0)$ & 32 \\
\hline 22 & 1,4-dioxane & 130 & $\mathrm{Pd}_{2}(\mathrm{dba})_{3}(5.0) / \mathrm{PCy}_{3}(20)$ & none & 0 \\
\hline 23 & 1,4-dioxane & 130 & $\mathrm{Pd}_{2}(\mathrm{dba})_{3}(5.0) / \mathrm{PCy}_{3}(20)$ & $\mathrm{KF}(5.0)$ & 0 \\
\hline 24 & 1,4-dioxane & 130 & $\mathrm{Pd}_{2}(\mathrm{dba})_{3}(5.0) / \mathrm{PCy}_{3}(20)$ & $\operatorname{AgF}(5.0)$ & 9 \\
\hline 25 & 1,4-dioxane & 130 & $\mathrm{Pd}_{2}(\mathrm{dba})_{3}(5.0) / \mathrm{PCy}_{3}(20)$ & CsF (10) & 55 \\
\hline $26^{c}$ & 1,4-dioxane & 130 & $\mathrm{Pd}_{2}(\mathrm{dba})_{3}(5.0) / \mathrm{PCy}_{3}(20)$ & $\mathrm{CsF}(10)$ & 53 \\
\hline 27 & DMF & 130 & $\mathrm{Pd}_{2}(\mathrm{dba})_{3}(5.0) / \mathrm{PCy}_{3}(20)$ & CsF (10) & 25 \\
\hline 28 & DME & 130 & $\mathrm{Pd}_{2}(\mathrm{dba})_{3}(5.0) / \mathrm{PCy}_{3}(20)$ & CsF (10) & 49 \\
\hline 29 & mesitylene & 160 & $\mathrm{Pd}_{2}(\mathrm{dba})_{3}(5.0) / \mathrm{PCy}_{3}(20)$ & CsF (10) & 21 \\
\hline 30 & DMSO & 130 & $\mathrm{Pd}_{2}(\mathrm{dba})_{3}(5.0) / \mathrm{PCy}_{3}(20)$ & $\mathrm{CsF}(10)$ & 0 \\
\hline 31 & toluene & 130 & $\mathrm{Pd}_{2}(\mathrm{dba})_{3}(5.0) / \mathrm{PCy}_{3}(20)$ & CsF (10) & 0 \\
\hline 32 & $\mathrm{MeCN}$ & 110 & $\mathrm{Pd}_{2}(\mathrm{dba})_{3}(5.0) / \mathrm{PCy}_{3}(20)$ & CsF (10) & 23 \\
\hline 33 & 1,4-dioxane & 110 & $\mathrm{Pd}_{2}(\mathrm{dba})_{3}(5.0) / \mathrm{PCy}_{3}(20)$ & CsF (10) & 17 \\
\hline
\end{tabular}


Table S1. Screening of Reaction Conditions (Continued) ${ }^{a}$

\begin{tabular}{llllll}
\hline entry & solvent & temp. $\left({ }^{\circ} \mathrm{C}\right)$ & $\mathrm{Pd}$ source $(\mathrm{mol} \%)$ & $\mathrm{F}^{-}$source (equiv) & yield $(\%)^{b}$ \\
\hline 34 & 1,4-dioxane & 90 & $\mathrm{Pd}_{2}(\mathrm{dba})_{3}(5.0) / \mathrm{PCy}_{3}(20)$ & $\mathrm{CsF}(10)$ & 1 \\
35 & $1,4-$ dioxane $^{d}$ & 130 & $\mathrm{Pd}_{2}(\mathrm{dba})_{3}(5.0) / \mathrm{PCy}_{3}(20)$ & $\mathrm{CsF}(10)$ & 24 \\
36 & $1,4-$ dioxane $^{e}$ & 130 & $\mathrm{Pd}_{2}(\mathrm{dba})_{3}(5.0) / \mathrm{PCy}_{3}(20)$ & $\mathrm{CsF}(10)$ & $79(80)^{f}$ \\
37 & $1,4-$ dioxane $^{g}$ & 130 & $\mathrm{Pd}_{2}(\mathrm{dba})_{3}(5.0) / \mathrm{PCy}_{3}(20)$ & $\mathrm{CsF}(10)$ & 56 \\
38 & $1,4-$ dioxane $^{h}$ & 130 & $\mathrm{Pd}_{2}(\mathrm{dba})_{3}(5.0) / \mathrm{PCy}_{3}(20)$ & $\mathrm{CsF}(10)$ & 67 \\
\hline
\end{tabular}

${ }^{a}$ Reactions were carried out on a $0.5 \mathrm{mmol}$ scale with $0.05 \mathrm{M}$ solution for $15 \mathrm{~h} .{ }^{b} \mathrm{NMR}$ yield estimated using 1,3,5-trimethoxybenzene as an internal standard. ${ }^{c}$ Reaction was carried out for $24 \mathrm{~h}$. ${ }^{d}$ The reaction was carried out with $0.01 \mathrm{M}$ solution. ${ }^{e}$ The reaction was carried out with $0.02 \mathrm{M}$ solution. ${ }^{f}$ The value in parentheses was isolated yield of 3aa. ${ }^{g}$ The reaction was carried out with $0.075 \mathrm{M}$ solution. ${ }^{h}$ The reaction was carried out with $0.1 \mathrm{M}$ solution.

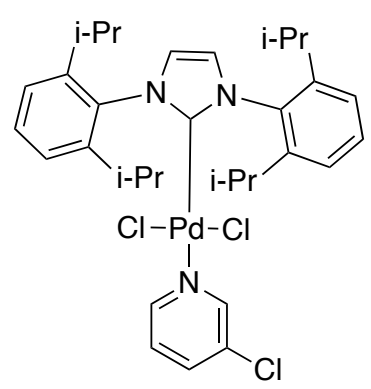

PEPPSI IPr

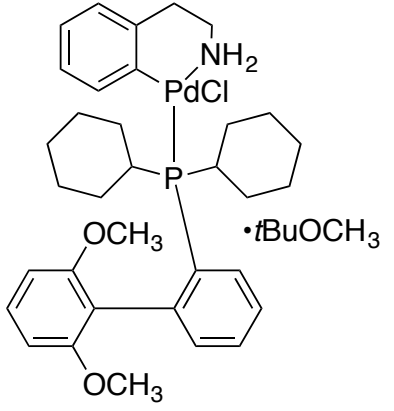

SPhos Pd G1<smiles></smiles>

XPhos Pd G1

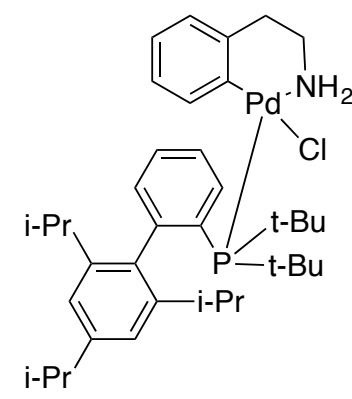

tBuXPhos Pd G1<smiles>COc1cc(P(O[Ge])(C2CCCCC2)(C2CCCCC2)C2CCCCC2)ccc1-c1c(OC)ccc(OC)c1-c1c(C(C)C)cc(C(C)C)cc1C(C)C</smiles>

BrettPhos Pd G3 

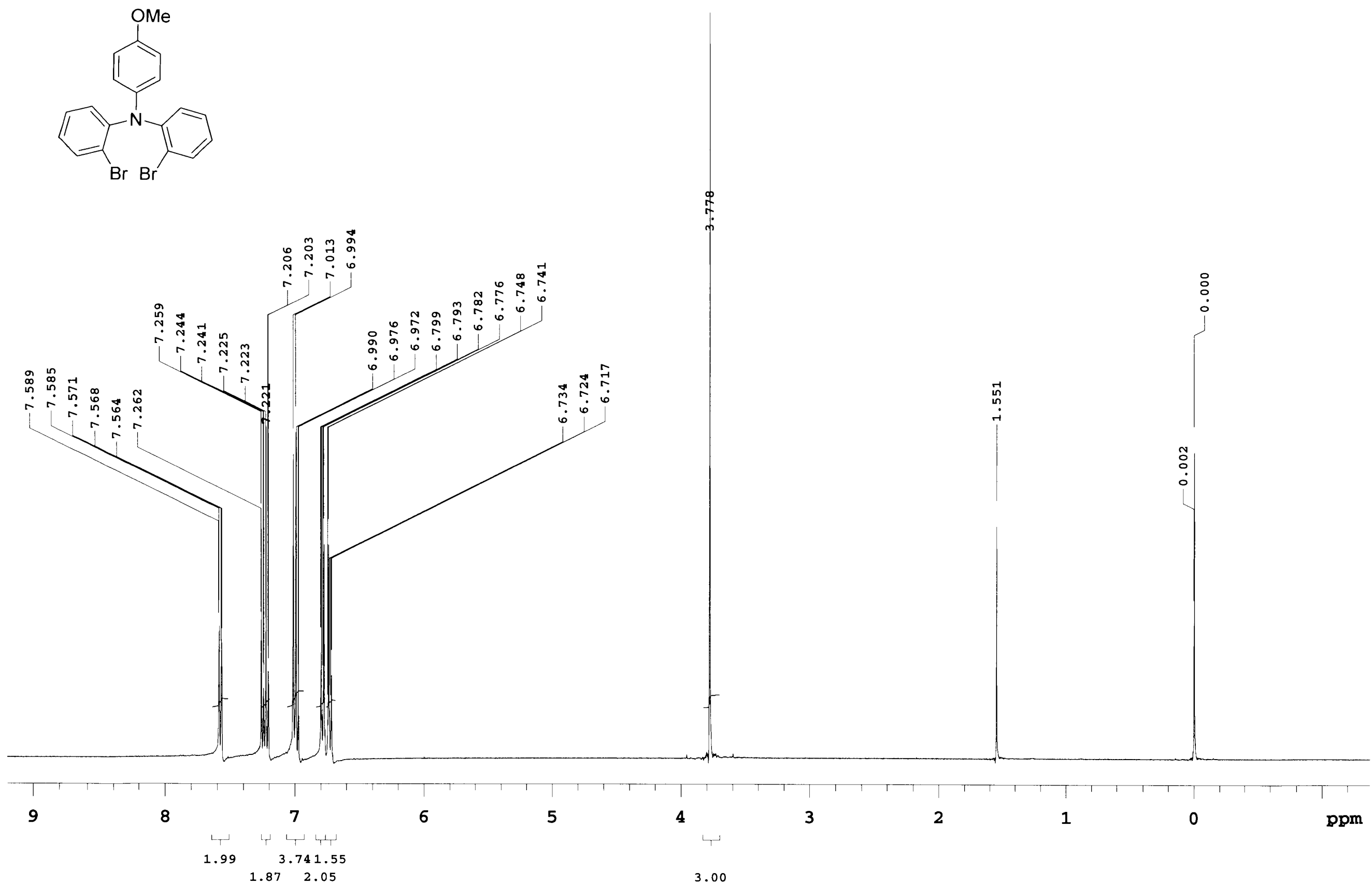

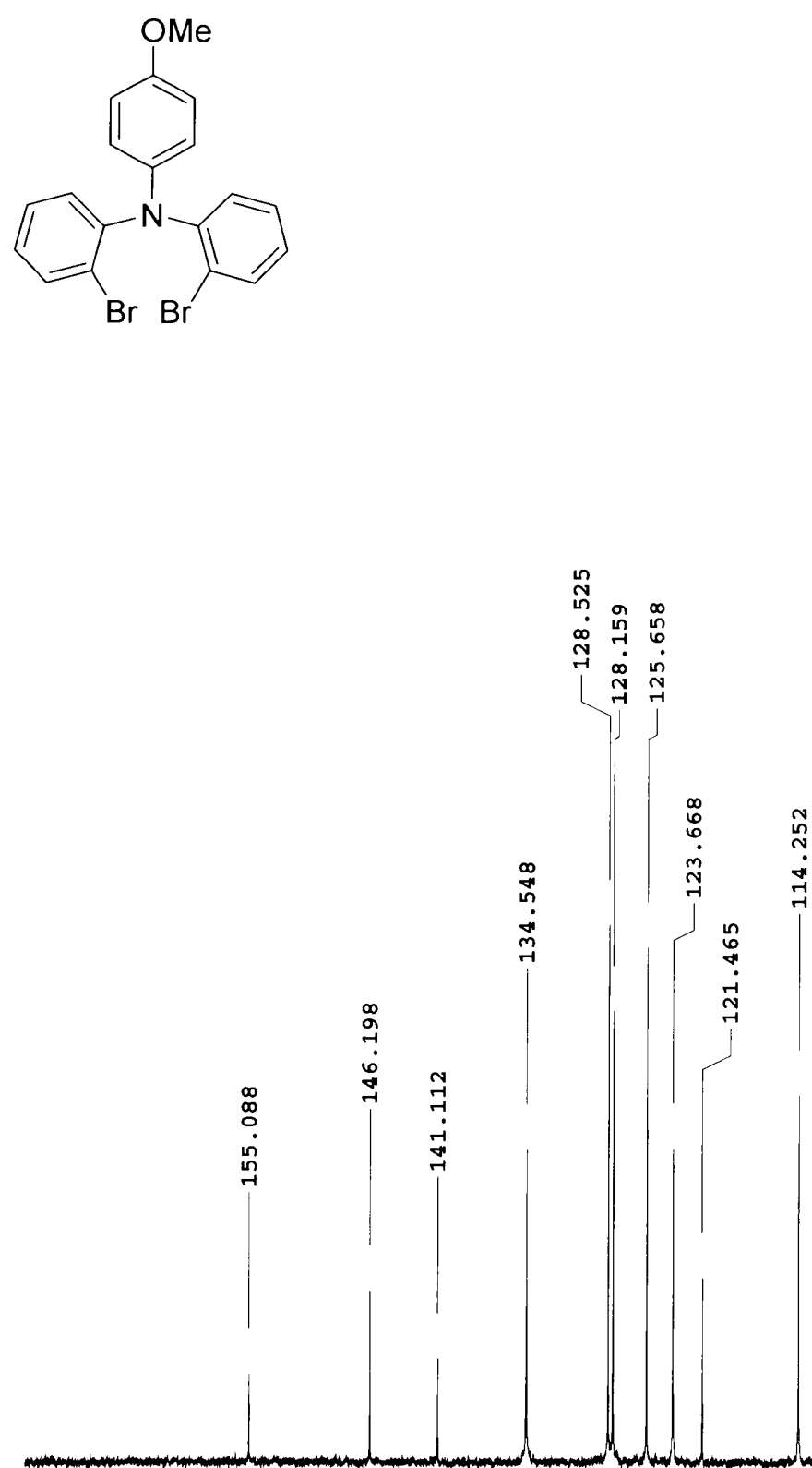

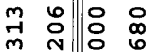

iิ

160

140

120

100

80

40

20

0 ppm 

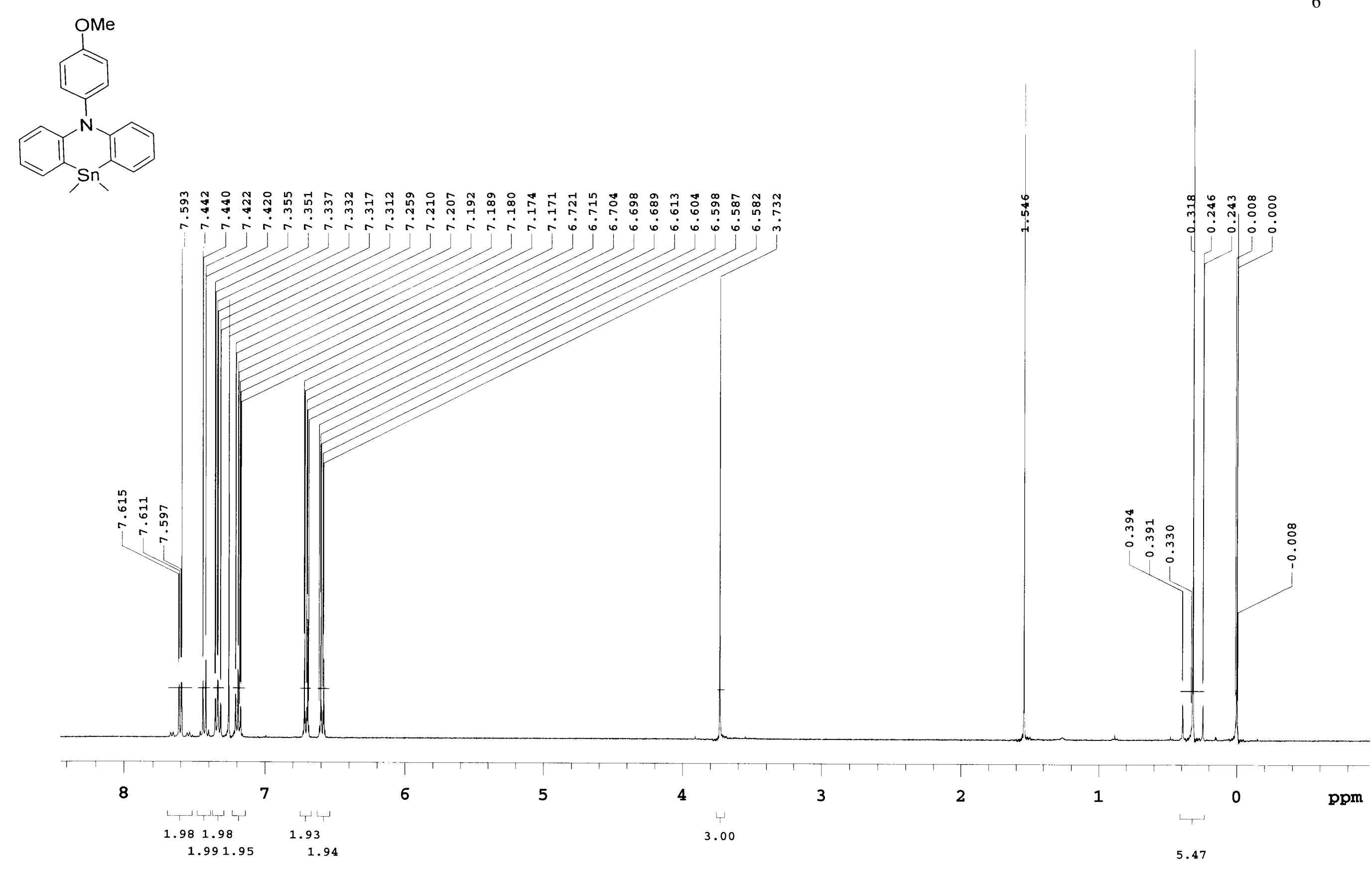

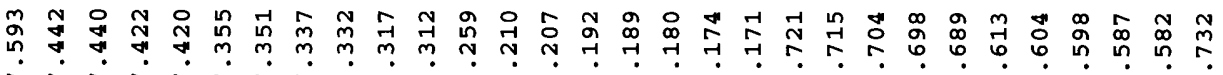



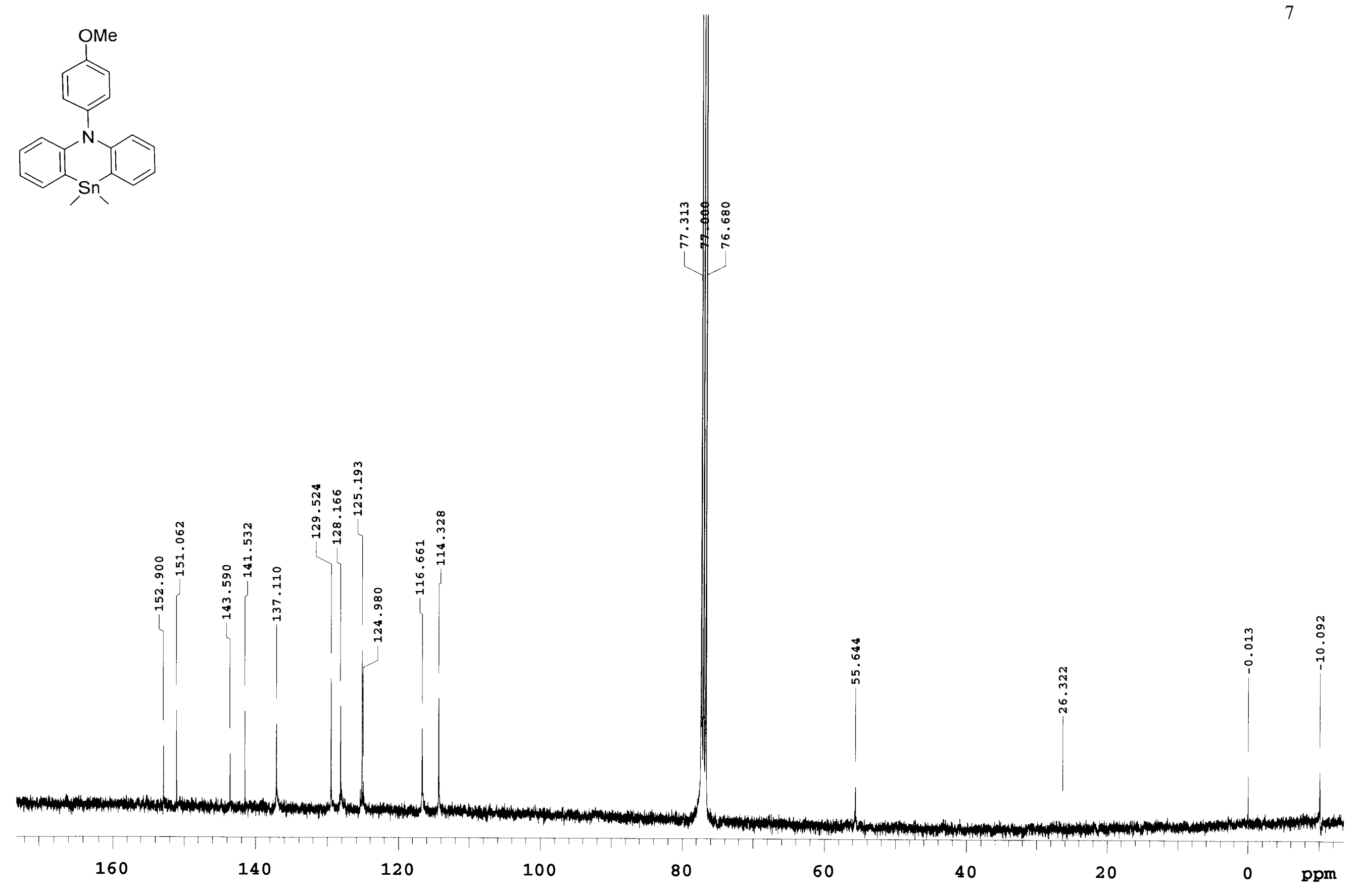
${ }^{1} \mathrm{H}$ NMR of $\mathbf{2 d}$

8<smiles>O=S1(=O)c2ccccc2C(=C(Br)Br)c2ccccc21</smiles>

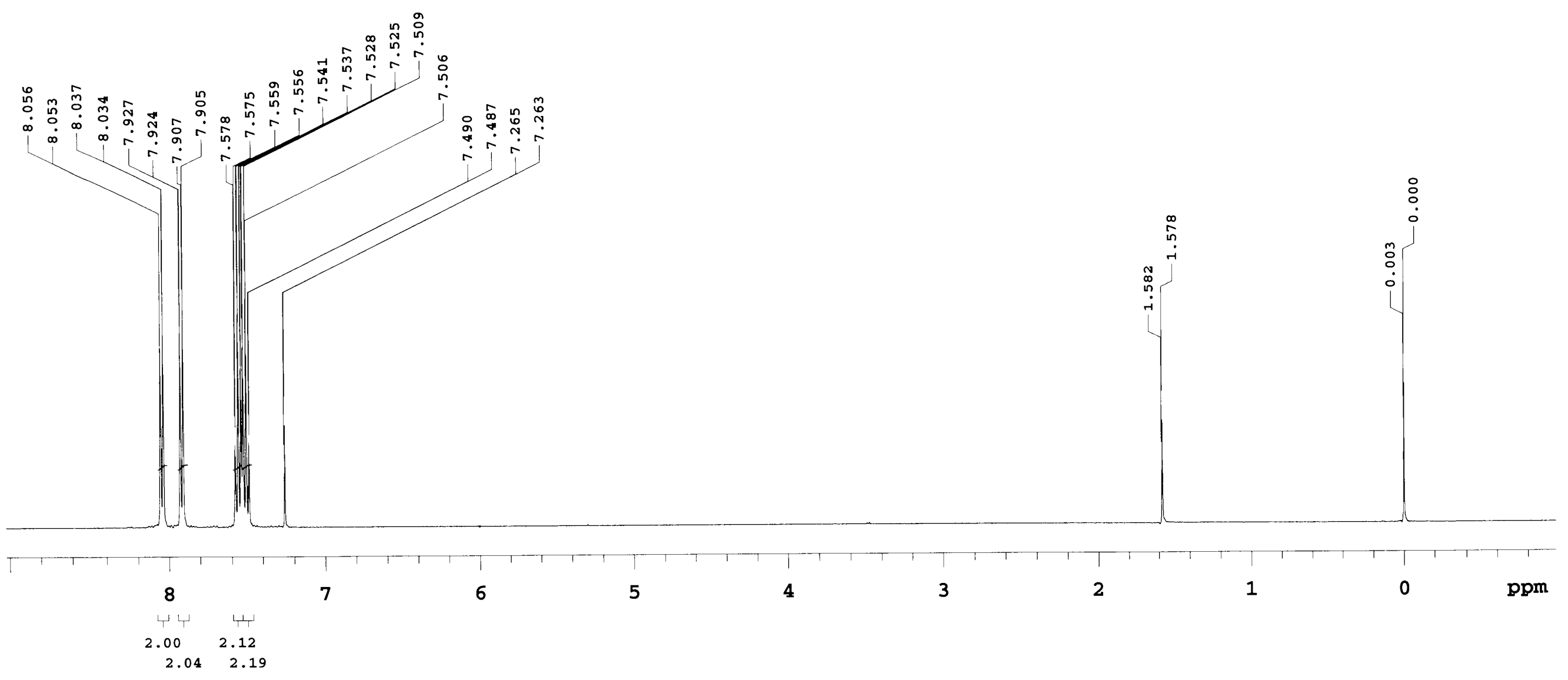



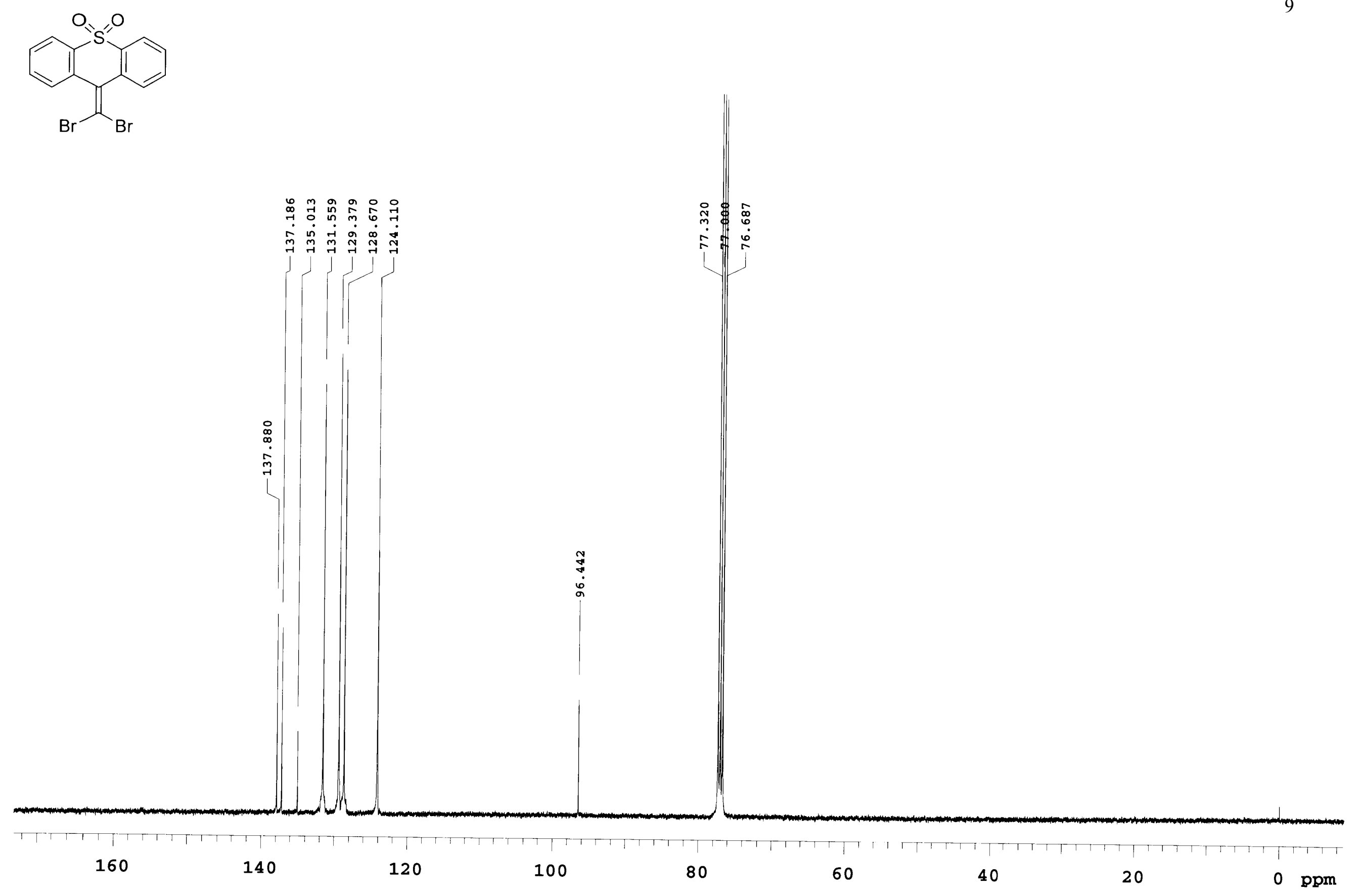


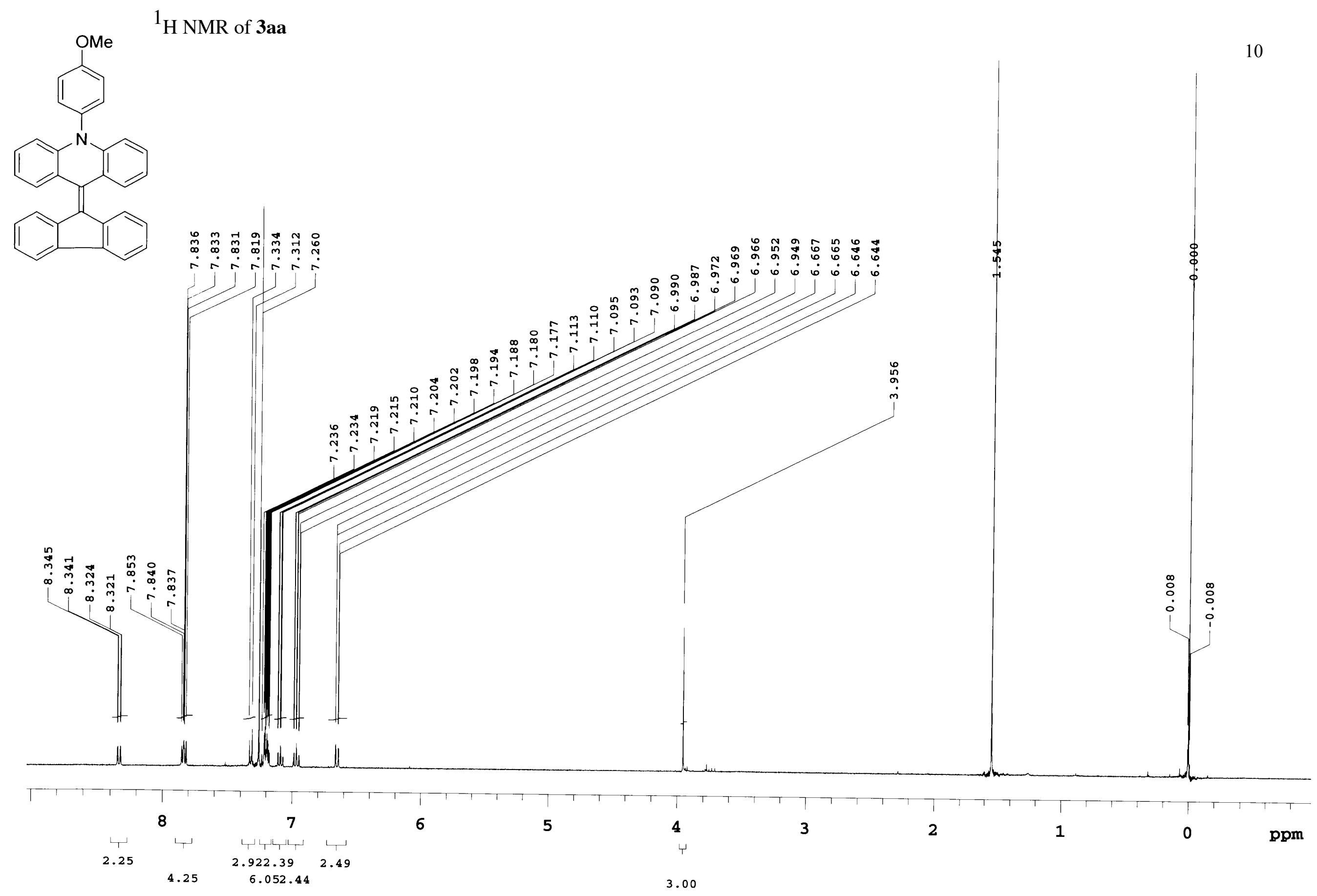



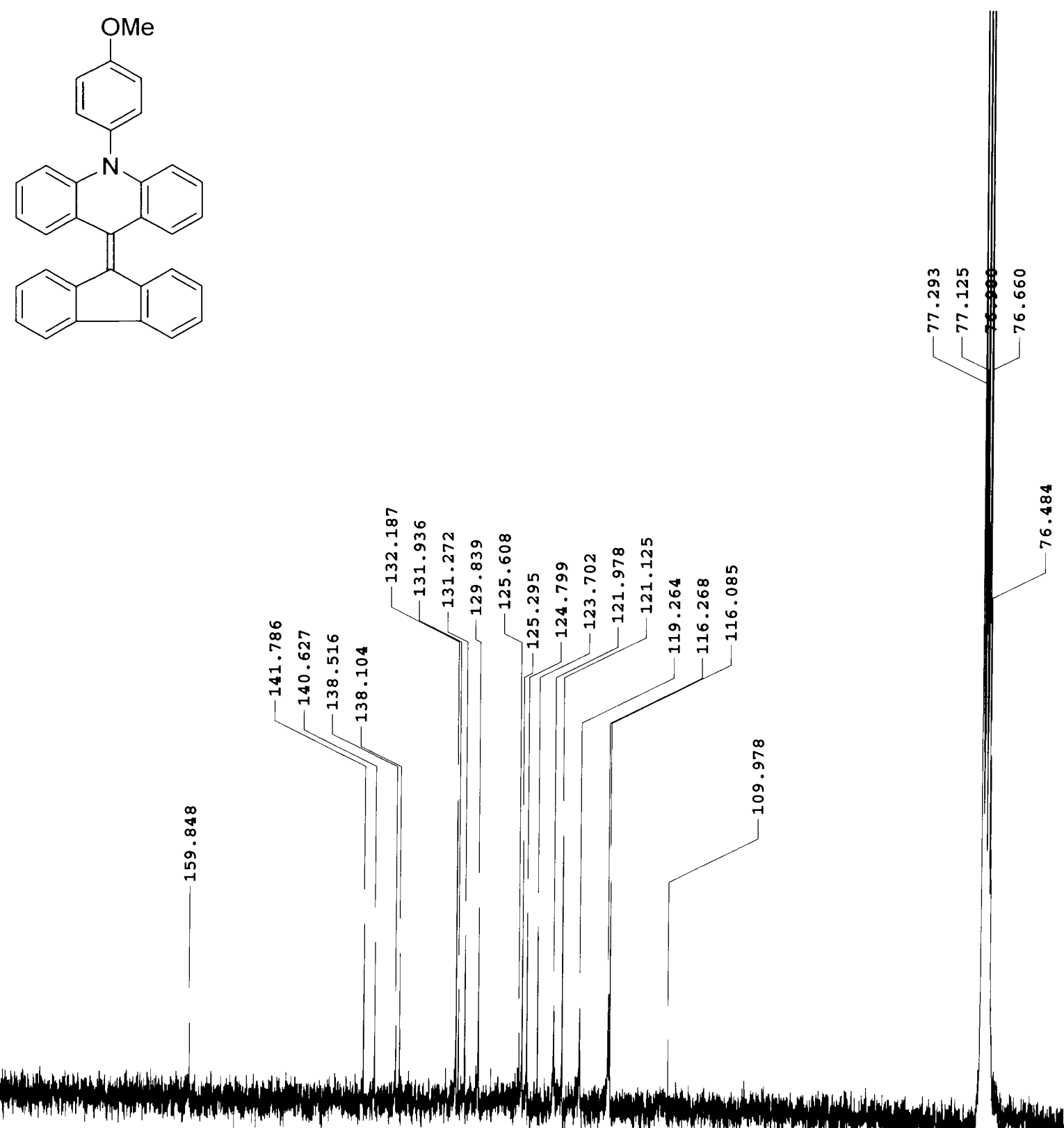

$\stackrel{8}{*}$

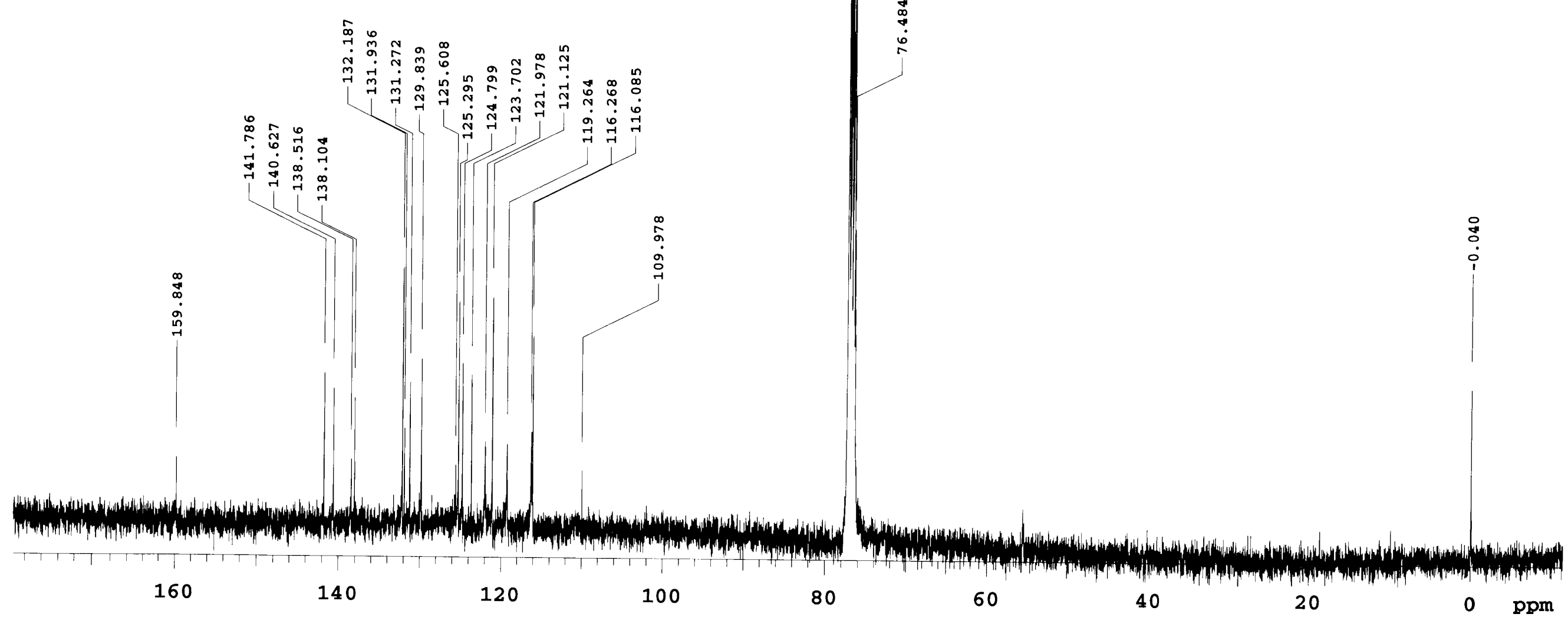




\section{${ }^{1} \mathrm{H}$ NMR of $\mathbf{3 a b}$}
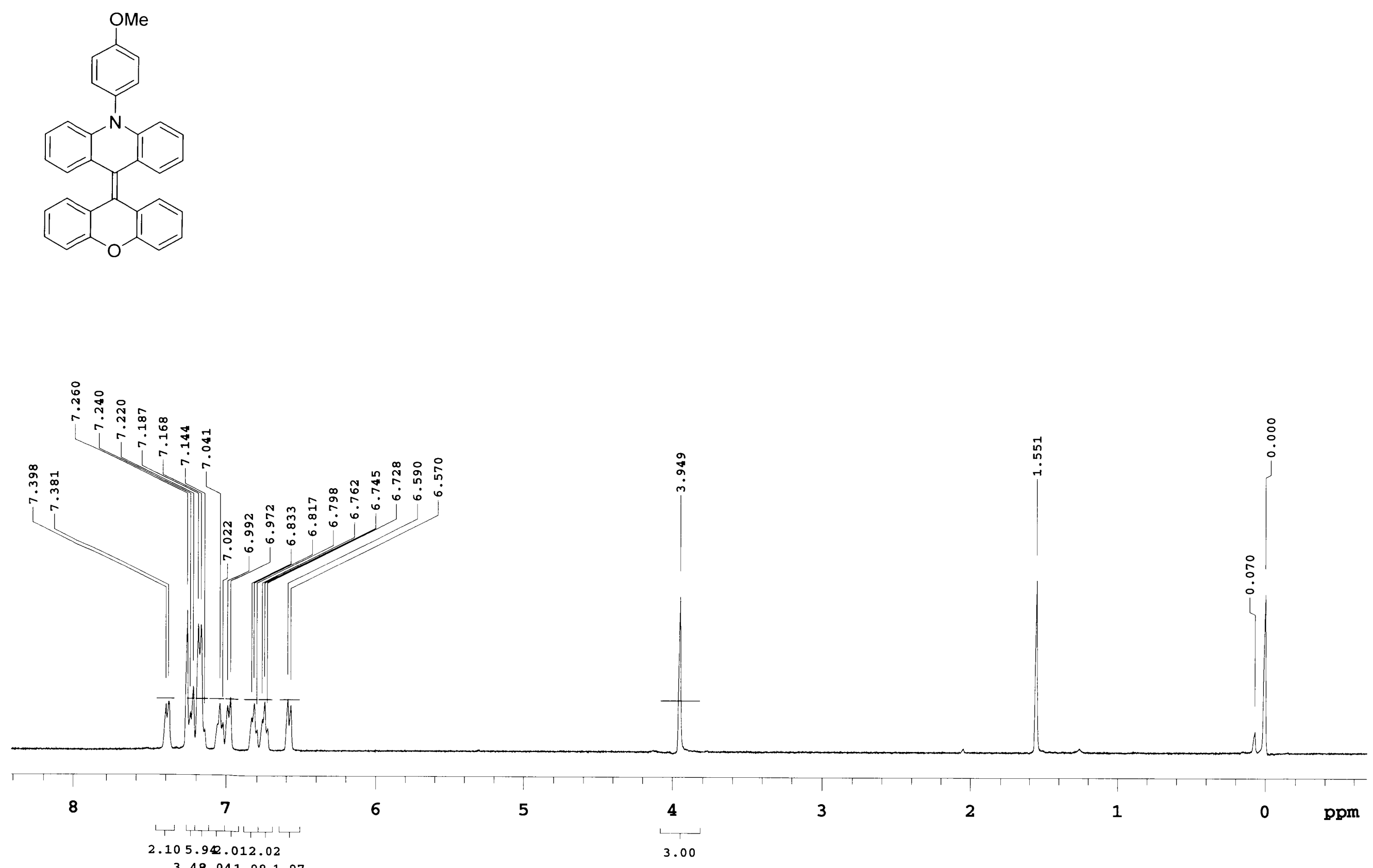


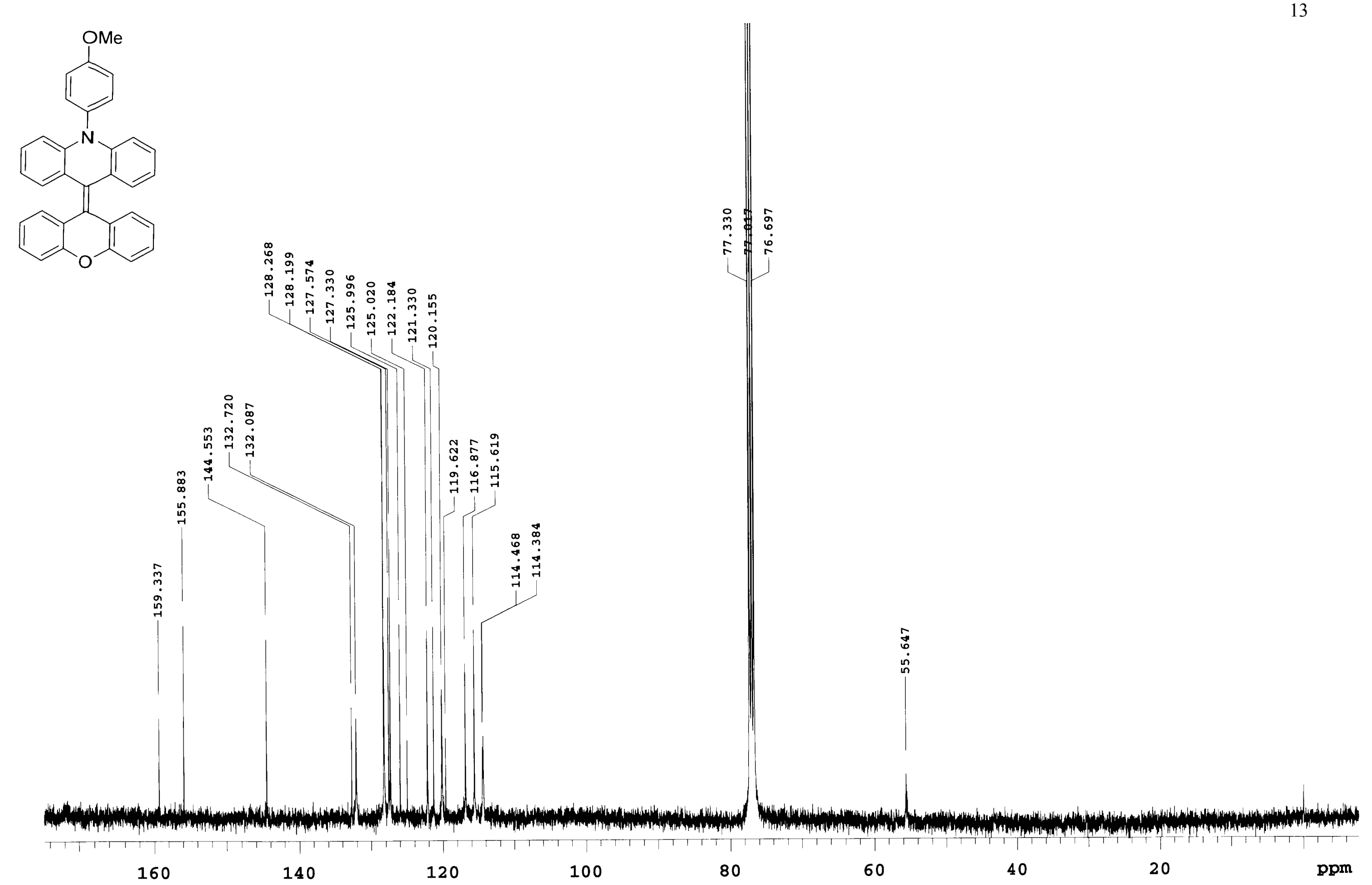




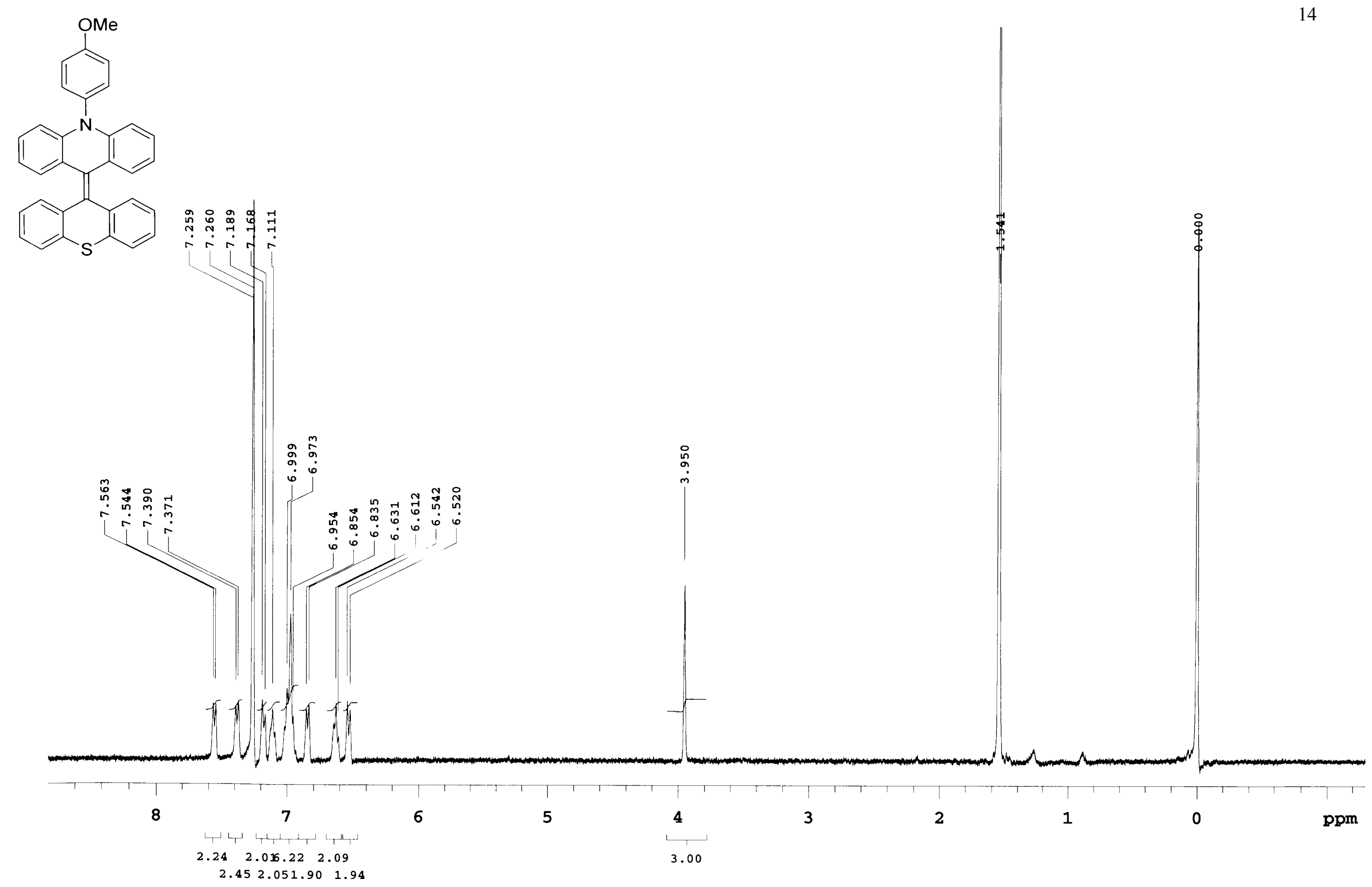



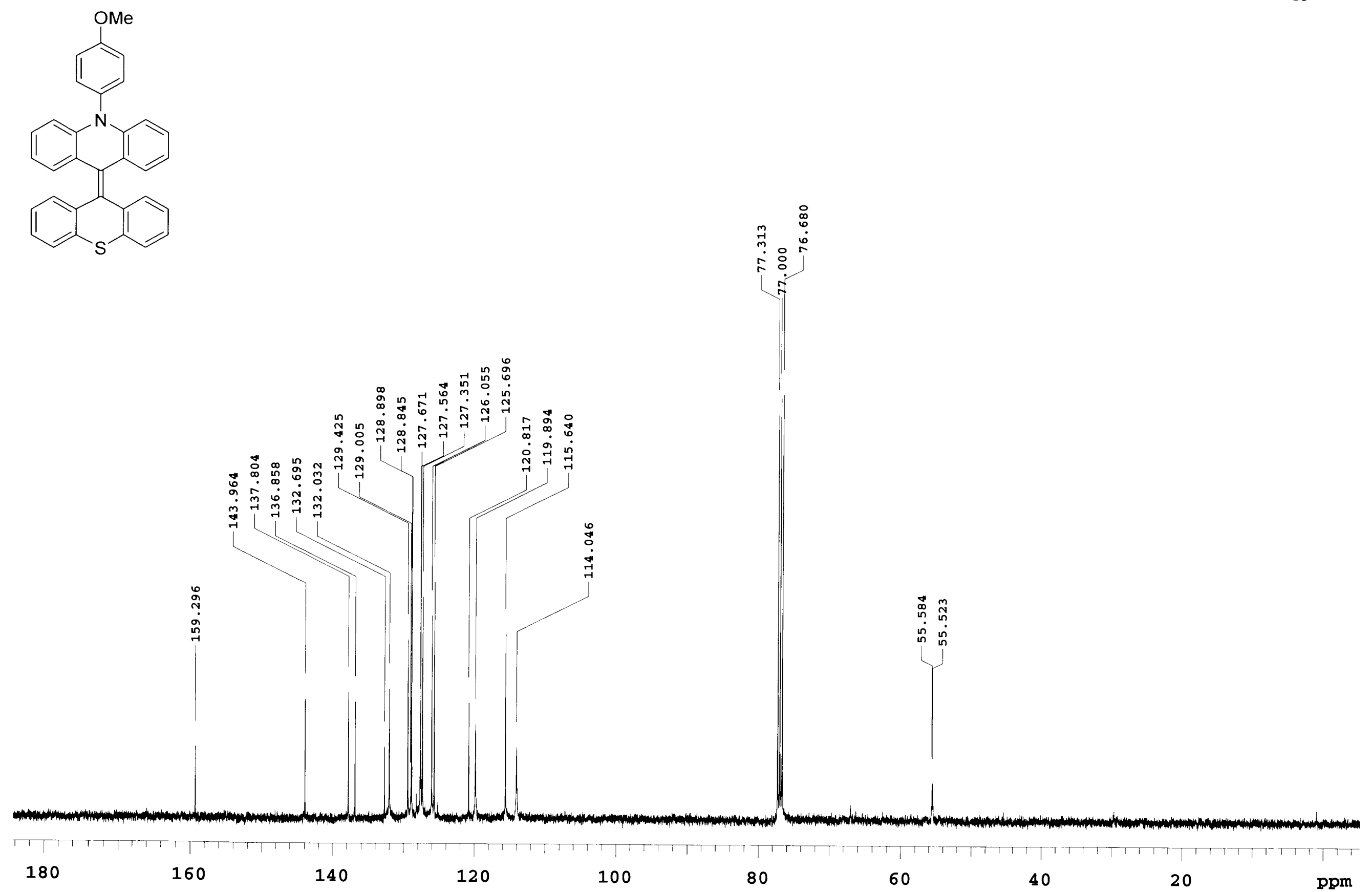


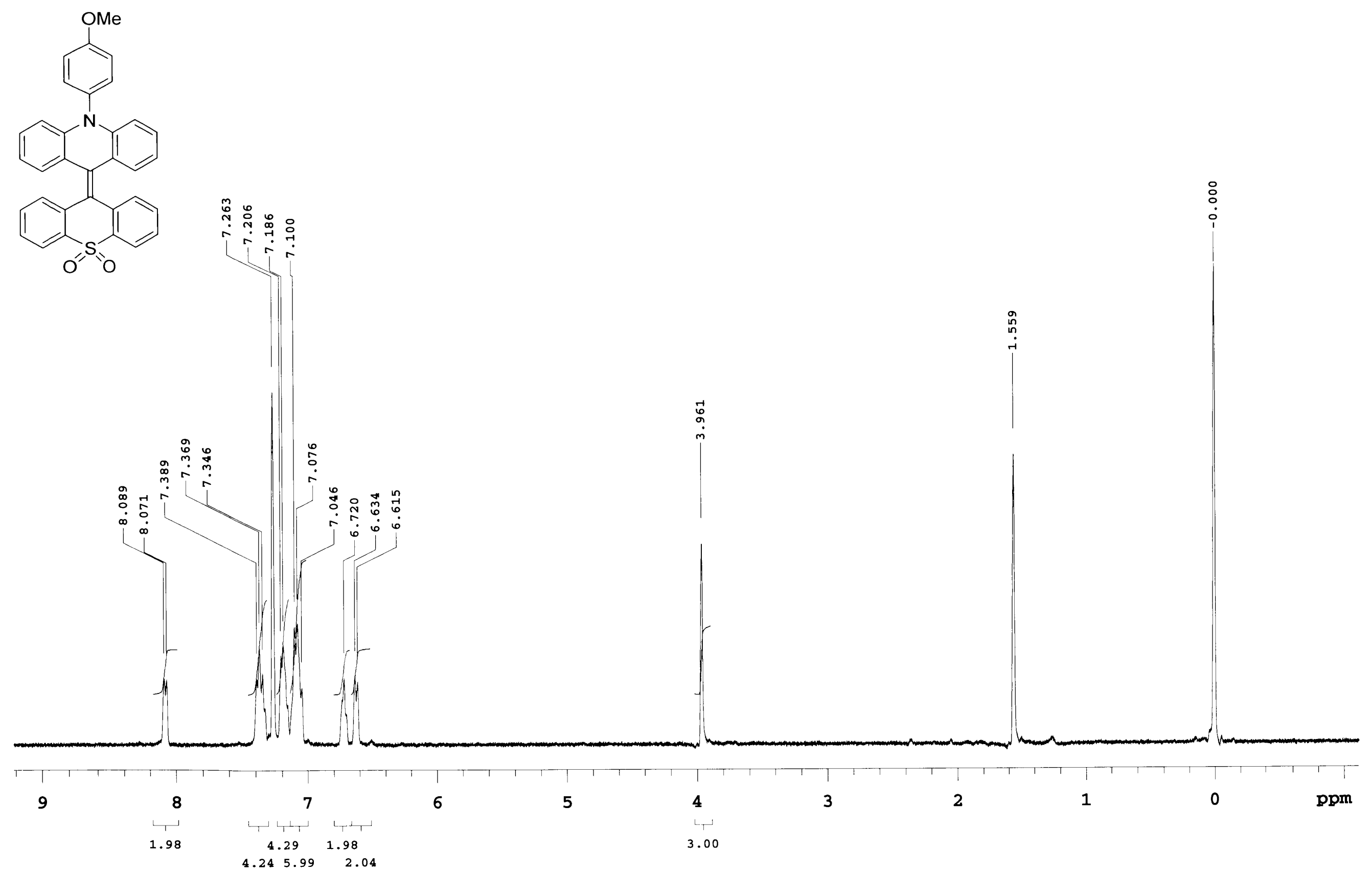



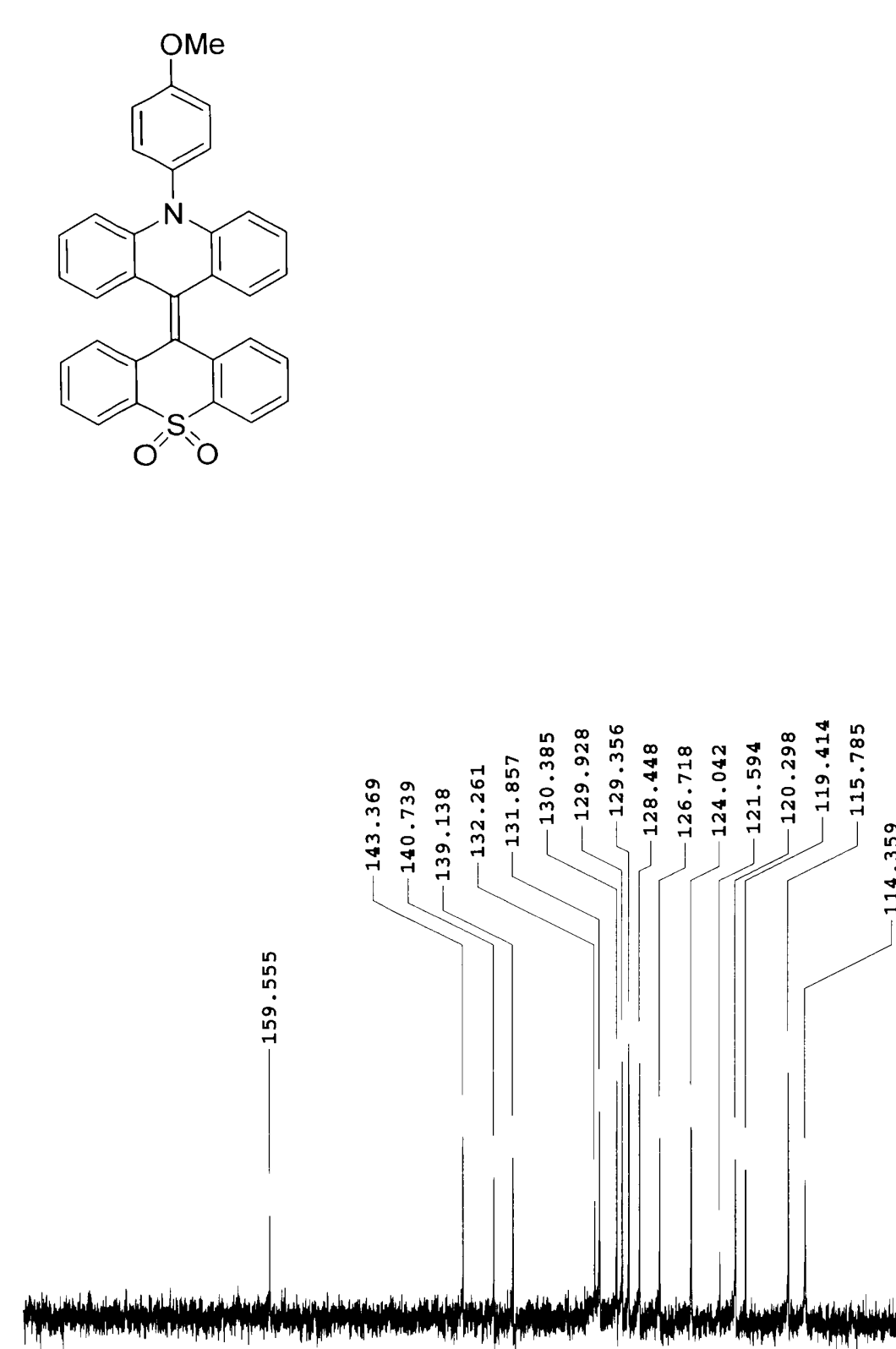

芴

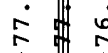

i

ชิ

咹㟧

i

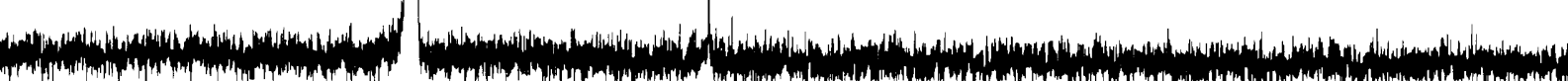

160

140

120

100

80

60

40

20

ppm 

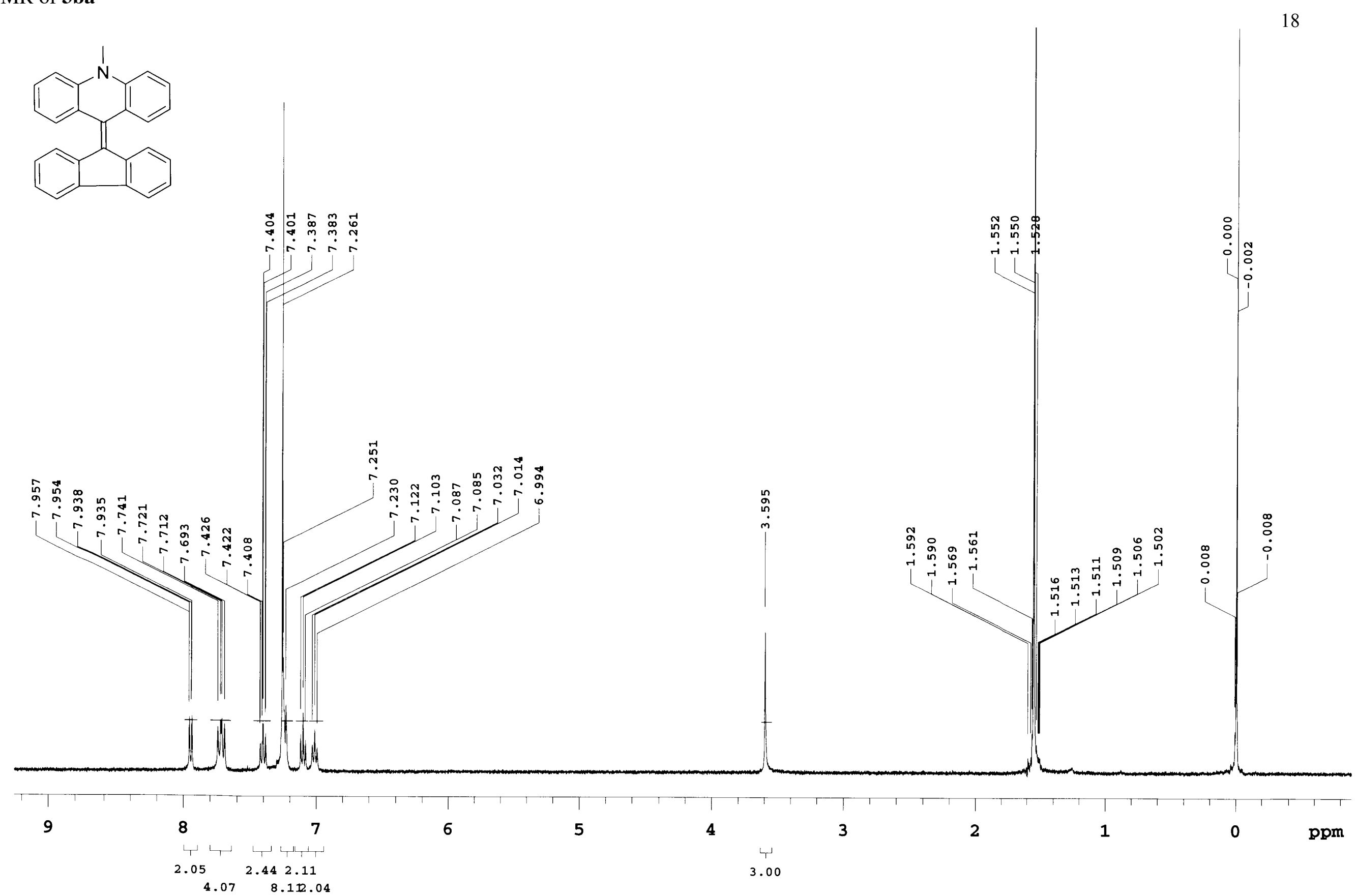

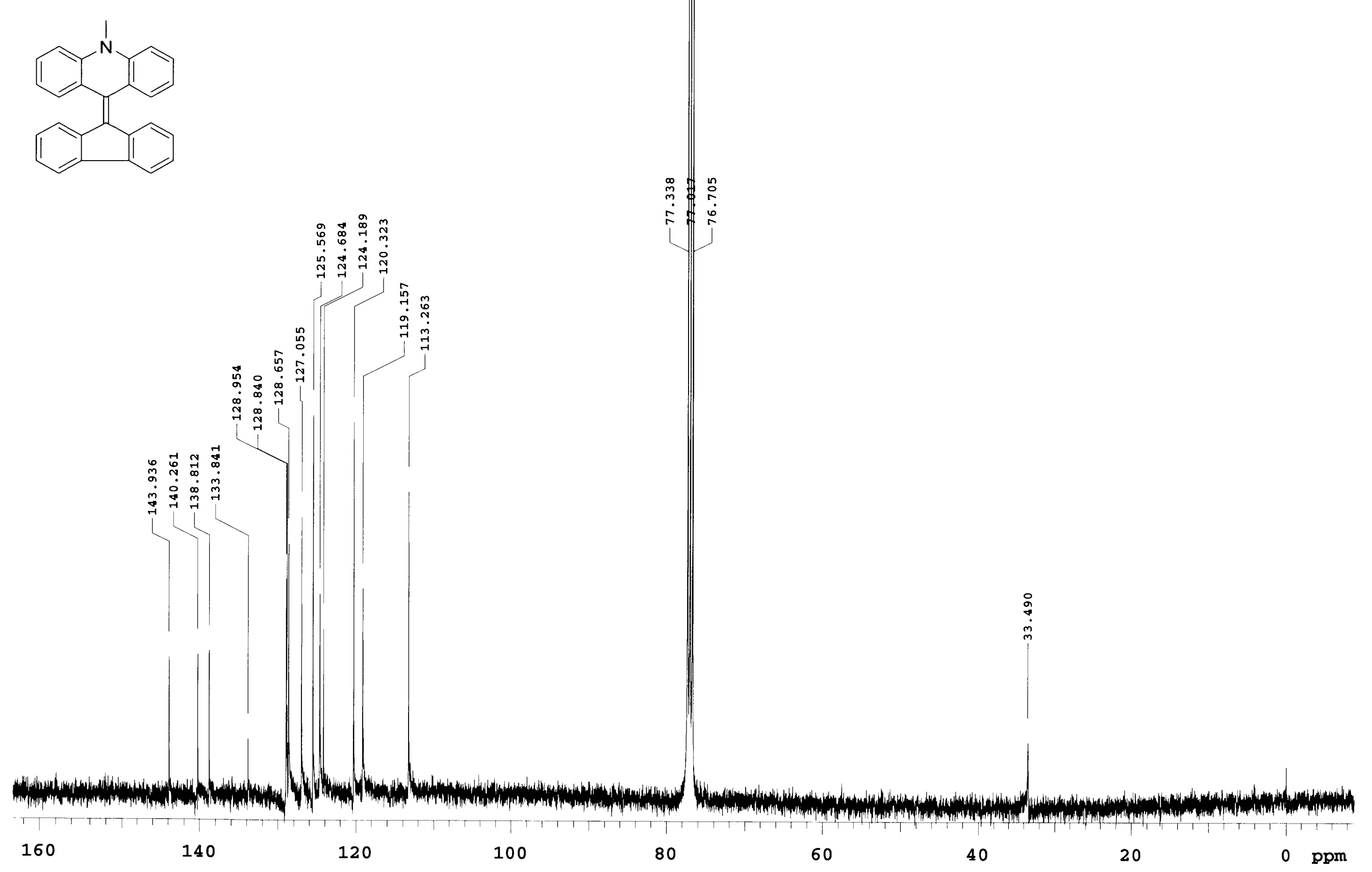

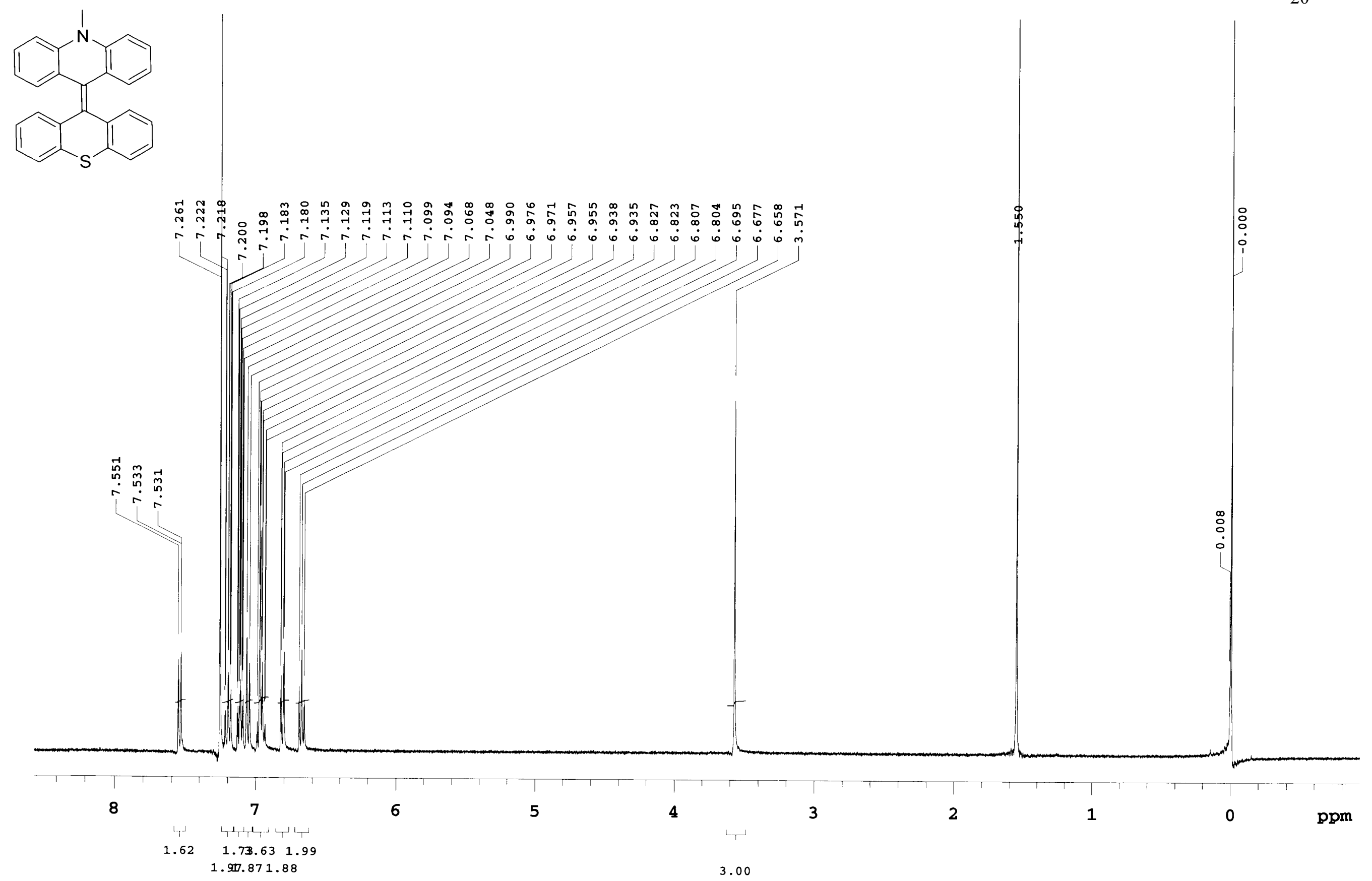

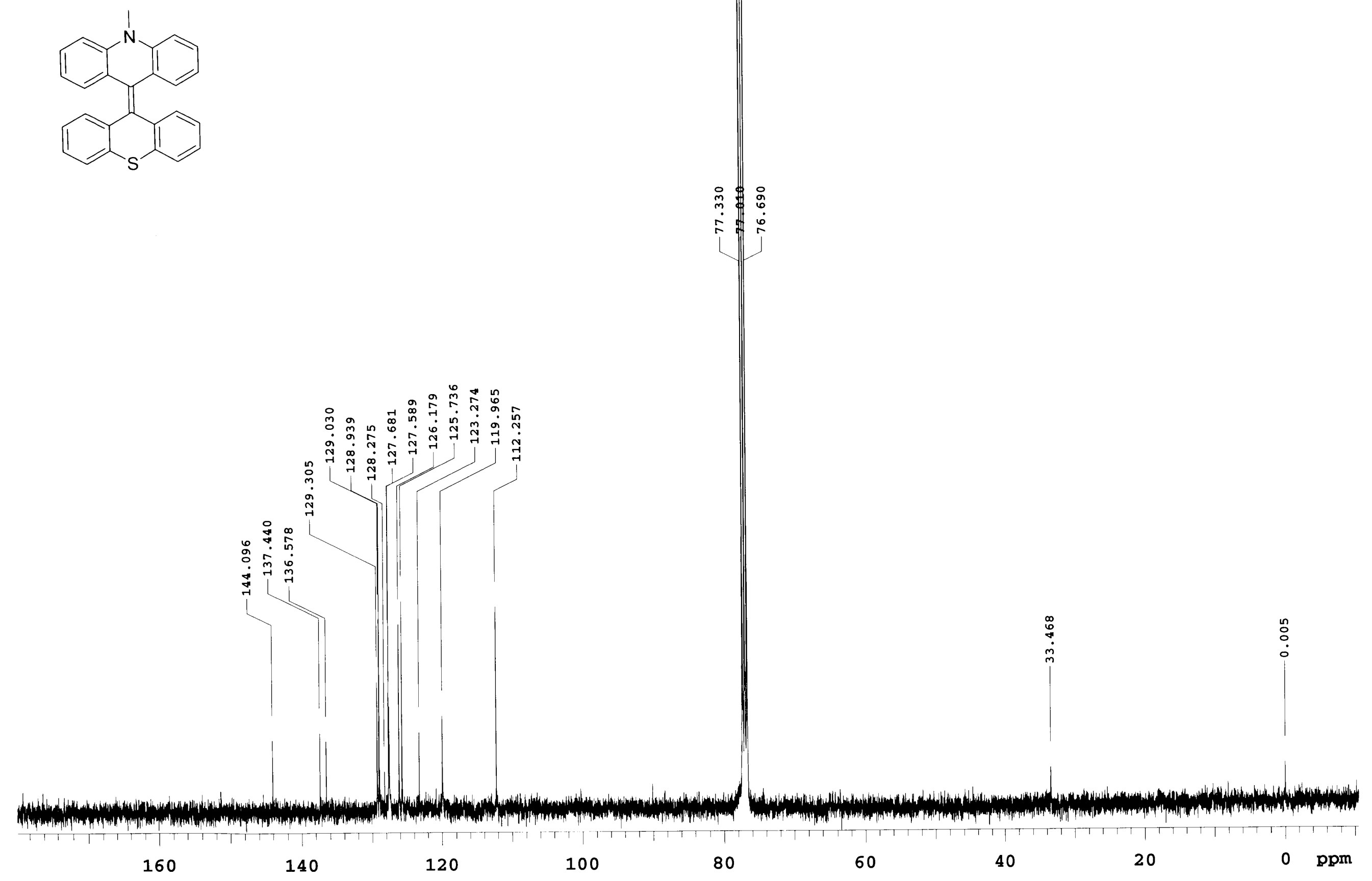

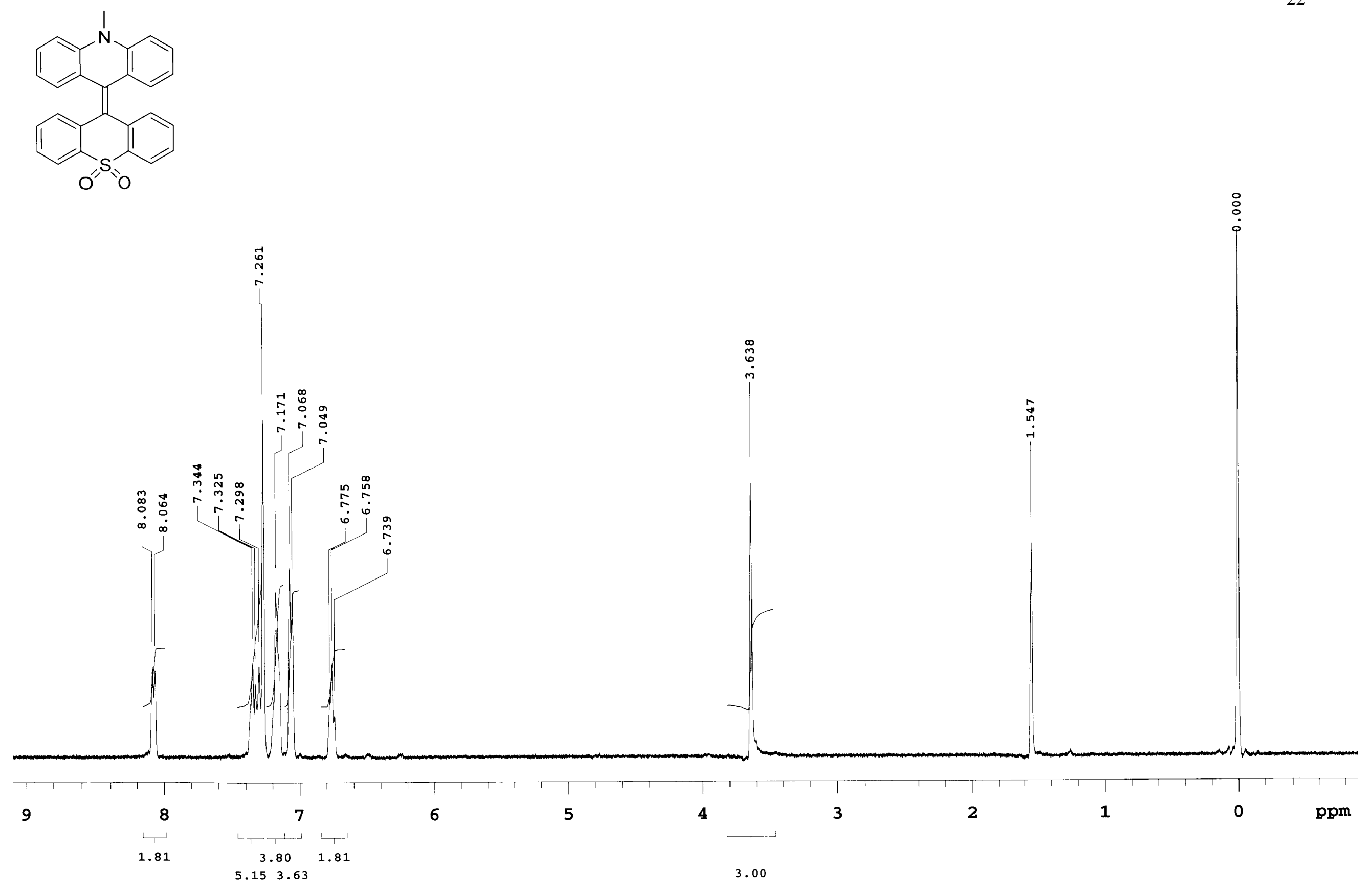

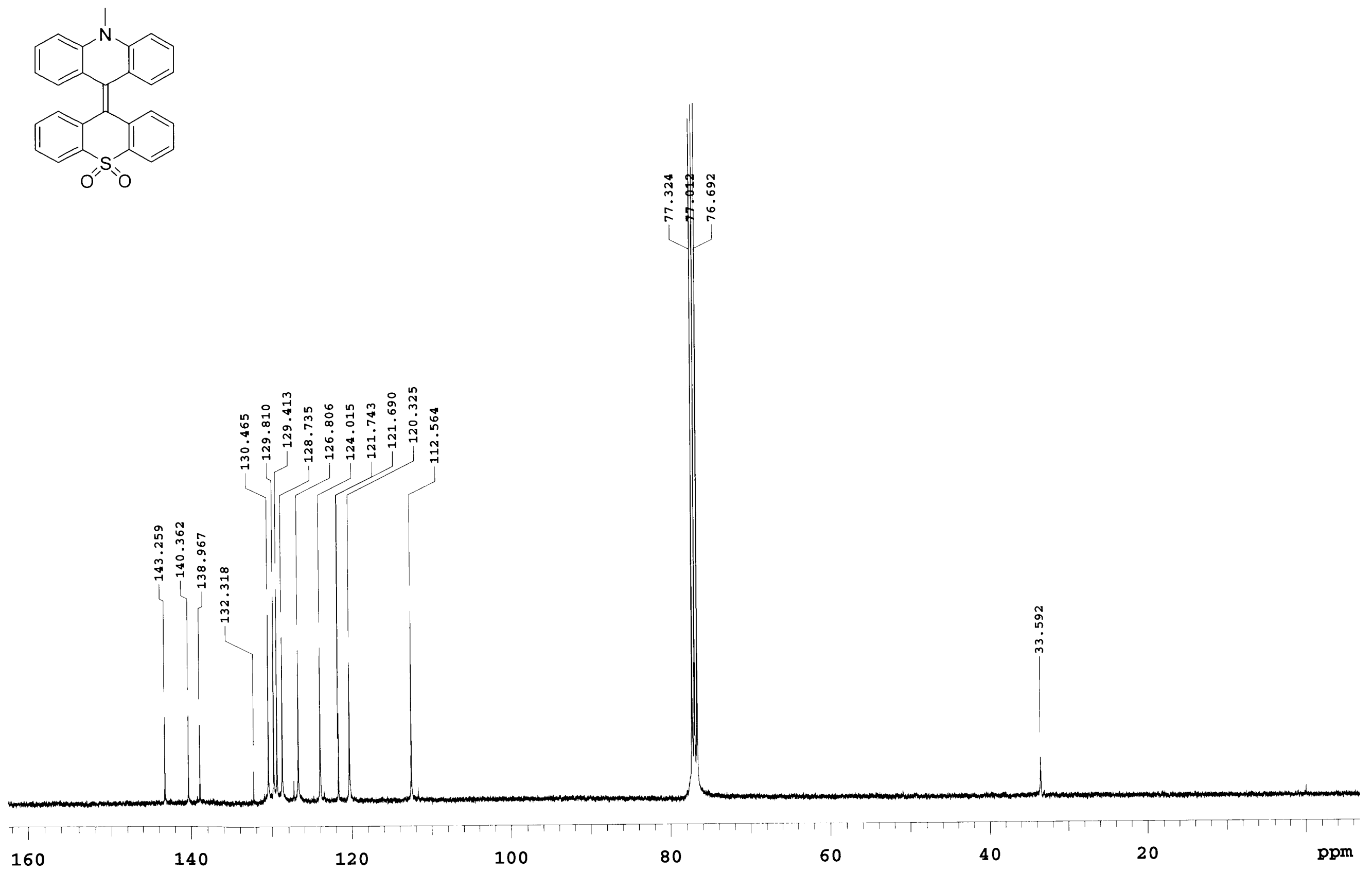